\title{
Factores geográficos en la epidemiología de la intoxicación por Karwinskia (tullidora) en México
}

\author{
Geographical factors in the epidemiology \\ of intoxication by Karwinskia (tullidora) \\ in Mexico
}

María Eugenia Arreola Nava 1

José Luis Vázquez Castellanos 2

Miguel Ernesto González Castañeda 3

\footnotetext{
1 Calle Miguel Galindo \# 2048, Jardines del Country, C.P. 44210, Guadalajara, Jalisco, México.

earreola@yahoo.com

2 Instituto Regional

de Investigación en Salud Pública, Departamento de Salud Pública, Centro Universitario de Ciencias de la Salud, Universidad de Guadalajara, Guadalajara, Jalisco, México. jolse@irisp.dsp.udg.mx

3 Departamento de Salud Pública, Centro Universitario de Ciencias de la Salud, Universidad de Guadalajara, Guadalajara, Jalisco, México. mgonzale@kock.mb.udg.mx
}

\begin{abstract}
Consumption of fruits from the genus Karwinskia may cause a flaccid paralysis that can be confused with the Guillan-Barré syndrome or poliomyelitis. Paralysis resulting from consumption of such fruit has emerged as a public health problem in certain regions of Mexico. The purpose of this study was to investigate geographical factors associated with this intoxication in the 72 cases reported in Mexico from 1990 to 1994. Location of cases coincides with the distribution of the 11 reported species of Karwinskia in the country. The majority of reported cases were related to the consumption of $\mathrm{K}$. humboldtiana, with a smaller number involving $\mathrm{K}$. mollis, $\mathrm{K}$. parvifolia, K. johnstonii, and K. rzedowskii. Most cases were located in regions with dry climates (79.2\%), arid vegetation (41.7\%), and altitudes under 1,000 meters above sea level (54.1\%). The study establishes three different risk areas: the Balsas river region in the southwestern central part of the country; the arid northern area; and the arid and dry central area. Cases were from rural communities with low levels of schooling and poor socioeconomic conditions
\end{abstract}

Key words Karwinskia; Poisoning; Paralysis

Resumen Las Parálisis Flácidas causadas por el consumo de los frutos de las plantas del género Karwinskia (tullidora) han emergido como un problema de salud pública en ciertas regiones de México. El objeto de este trabajo fue investigar los factores geográficos asociados a esta patología, en 72 casos reportados en México de 1990 a 1994. La distribución geográfica de casos coincide con las 11 especies reportadas de Karwinskia en México. La mayoría se relacionaron con la $\mathrm{K}$. humboldtiana y, en menor medida, con K. mollis, K. parvifolia, K. johnstonii y K. rzedowskii. La presencia de casos es mayor en regiones con climas secos (79,2\%), vegetación de Matorrales Aridos $(41,7 \%)$ y altitudes menores de mil metros (54,1\%). Se determinaron tres diferentes zonas de riesgo: la zona del río Balsas, la región norte del país, así como las zonas áridas y secas centrales de los estados de Puebla, Hidalgo, San Luis Potosí y Querétaro. Las comunidades menores de 2.500 habitantes y con niveles de bienestar y de educación bajos son las más afectadas.

Palabras clave Karwinskia; Intoxicación; Parálisis 


\section{Introducción}

En México, a medida que la poliomielitis ha desaparecido, otras patologías anteriormente poco valoradas como problemas de salud pública han emergido como causas de parálisis. Tal es el caso de las parálisis flácidas producidas por la intoxicación con una planta del género Karwinskia, cuyo nombre común es "tullidora", de la que se tienen noticias desde tiempos coloniales y que continuamente son reportadas en México (Castillo-Nájera,1918; Padron, 1951; Carrada-Bravo et al., 1983; Puértolas et al., 1984).

Entre 1980 y 1989, la Dirección General de Epidemiología registró 94 brotes de intoxicación por Karwinskia. A partir de 1990 se han seguido reportando dentro del Sistema de Vigilancia de Parálisis Flácidas Agudas (PFA) casos aislados, y algunos brotes de pequeña magnitud.

El objetivo de este trabajo es conocer los factores geográficos que tienen relación con los casos de intoxicación por Karwinskia en la República Mexicana.

El género Karwinskia incluye unas 15 especies para América, cuyo hábitat natural se extiende de las regiones áridas del sur de los Estados Unidos de América a México, Centroamérica, norte de Colombia, Cuba, Haití y República Dominicana (Fernández, 1993). Se caracteriza por ser árboles o arbustos rígidos, entre 1 y 8 metros de altura. El fruto es de cáscara fibrosa, carnoso y de hueso fuerte y leñoso con una semilla. Su forma es subglobosa; de color violeta oscuro y de sabor agradable. En México se han encontrado 11 especies de este género, las cuales son: K. calderonii, K. humboldtiana, K. johnstonii, K. mollis, K. latifolia, K. parvifolia, K. rzedowskii, K. subcordata, K. tehuacana, K. umbellata y K. venturae. Algunos de los nombres comunes con que se conocen a las diferentes especies en México son: tullidora, coyotillo, margarita, capulincillo, cacachila, tuvii, pimientillo (Fernández, 1992).

Las sustancias tóxicas de la planta se encuentran en las semillas y se disuelven fácilmente en la saliva. Estas se ligan a la albúmina de la sangre y poco a poco se van liberando, por lo que su efecto puede ser acumulativo, de ahí que la parálisis aparezca semanas o meses después de la ingestión del fruto (Bermúdez, 1989). Las toxinas de la Karwinskia afectan las fibras periféricas de las neuronas motoras. Su acción específica es sobre las células de Schwann o neurolemocitos, cuya función es la de mantener la conducción del impulso nervioso así como de aislar y proteger al axón (Hernández \& Muñoz, 1982; Bermúdez et al., 1995).
La intoxicación por frutos de Karwinskia se manifiesta como una parálisis motora afebril, ascendente, progresiva y simétrica que puede afectar los músculos intercostales y llevar a la muerte (Puértolas et al., 1984; Bermúdez, 1989). Frecuentemente el cuadro se confunde con el Síndrome de Guillain-Barré y con lesiones del asta anterior de la médula espinal como la Poliomielitis (Escobar \& Nieto, 1965; Hernández \& Muñoz, 1984; Garza et al., 1992).

Se han determinado cuatro compuestos tóxicos presentes en la mayoría de las especies de Karwinskia (Waksman et al., 1989; Fernandez, 1992; Garza et al., 1992):

a) 7- [3'-4' -dihidro-7' -dimetoxi-1-1' -3' -dimetil-10'-hidroxi-1' H-naftol (2',3'-C') 5-piranil] 3,4-dihidro-3-metil-3,8,9-trihidroxi-1(2H)antracenona; compuesto que, de acuerdo a su peso molecular, se ha denominado T-544, responsable de los efectos neurológicos, esto es, afectación de los axones motores, desmielinización de las células de Schwann.

b) 2,3-dimetil-3,3',8,8',9'-hexahidroxi-3,3', 4,4'-tetrahidro (7,10' -biantraceno)-1,1' $(2 \mathrm{H}$, 2'H)-diona; llamado T-514, responsable de las lesiones pulmonares, del hígado y del miocardio.

c) 3,4-dihidro-3,3-dimetil-1',3,8,8',9-pentahidroxi(7,10-biantraceno)-1,9(2H,10'H)-diona; denominado T-496, y que únicamente produce diarrea.

d) 7-(2'-acetil-6',8' -dimetoxi-3'-metil-1'-hidroxinaft-4' -il)-3,4-dihidro-3-metil-3,8,9-trihidroxi-1(2H)-antracenona; también conocido como T-516, y que, hasta el momento, no se sabe con certeza su actividad específica.

\section{Metodología}

Se utilizó la base de datos del programa de vigilancia epidemiológica de PFA de 1990 a 1994. Los datos se obtuvieron de los estudios epidemiológicos de PFA mediante un formulario diseñado para tal fin. La detección de toxina en sangre se hizo mediante el método de cromatografía de capa fina en la Universidad Autónoma de Nuevo León. La identificación botánica fué realizada en el Herbario Nacional de la Universidad Nacional Autónoma de México UNAM y en el Instituto de Botánica de la Universidad de Guadalajara. Las informaciones cartográfica y monográfica sobre las variables físico-geográficas y socioeconómicas se obtuvieron del Instituto Nacional de Estadística, Geografía e Informática (INEGI). 


\section{Resultados}

Las plantas de este género se encuentran como miembros de vegetaciones de Bosque Tropical Caducifólio, Bosque de Encino, Matorral Xerófilo, Bosque de Juníperos, Pastizal, Bosque Tropical Perenifólio, así como de la vegetación secundaria, es decir, resultante a la desaparición de los anteriores conjuntos vegetales (Tabla 1). El género es de amplia distribución geográfica, ya que se localiza desde la Península de la Baja California hasta la Península de Yucatán. Sólo en cuatro Entidades no se ha reportado hasta el momento la presencia de Karwinskia: Baja California Norte, Tlaxcala, Tabasco y el Distrito Federal.

La K. humboldtiana es la de más amplia distribución. En zonas más restringidas en el occidente, centro y sureste del país, se desarrollan las otras especies (Figura 1). La mayor concentración de especies y de plantas se da en las partes centro y sur del país.

Los estados con mayor número de especies son Guerrero, con cinco (K. calderonii, K. humboldtiana, K. johnstonii, K. umbellata, K. venturae); Puebla, con cuatro (K. humboldtiana, K. mollis, K. tehuacana, K. umbellata) y, con tres especies cada uno, los estados de Jalisco (K. humboldtiana, K. latifolia, K. rzedowskii); Querétaro (K. humboldtiana, K. mollis, K. subcordata); Sinaloa (K. humboldtiana, K. parvifolia, K. latifolia) y Zacatecas (K. humboldtiana, K. latifolia, K. rzedowskii).

Se revisaron 72 casos de PFA por consumo de Karwinskia, de los cuales 55,6\% fueron en el sexo masculino. El $68,1 \%$ de casos se presentaron en menores de cinco años, seguido por el grupo de cinco a nueve años con el 25\%; en conjunto, estos dos grupos de edad representan el 93,1\% de los casos (Figura 2). En el 83,3\% de los casos, se obtuvo el antecedente de haber ingerido los frutos. De los casos que cuentan con la información, el tipo de ingesta única es el de mayor porcentaje $(6,9 \%)$, aunque la de tipo intermitente es la segunda en importancia $(4,2 \%)$. En 44 de los casos, se realizó la detección de toxinas de Karwinskia en sangre, y 40 resultaron positivos.

Las especies identificadas con mayor frecuencia en la intoxicación fueron K. humboldtiana $(22,2 \%), K$. mollis y $K$. parvifolia (4,2\%, cada una) y K. johnstonii y $K$. (1,4\%, cada una). La mayoría de los pacientes intoxicados $(61,1 \%)$ presentaron parálisis de las cuatro extremidades. En el 30,6\% se afectaron únicamente los miembros inferiores. El mayor porcentaje $(56,9 \%)$ se presentó en el primer trimestre del año. En la Figura 3, destacan los meses de febrero y marzo como los de la máxima ocurrencia. Los estados que reportaron el mayor número de intoxicaciónes fueron Guerrero, con $14(19,4 \%)$, seguido por Nuevo León, con nueve $(12,5 \%)$, San Luis Potosí, con ocho $(11,1 \%) y$, en igual porcentaje, los estados de Tamaulipas, Querétaro y Guanajuato, cada uno con siete casos $(9,7 \%)$ (Figura 1$)$.

Entre los tipos de vegetación, destacan dos, los tipos de Matorral Árido y Semiárido, asociados al $41,7 \%$ de los casos. Asociadas a este tipo

Tabla 1

Características botánicas y geográficas de las especies de Karwinskia en México.

\begin{tabular}{|c|c|c|c|c|}
\hline Nombre & Floración & Frutos & Tipos de vegetación* & Altitud \\
\hline K. calderonii & Jun-Ags & Sep-Dic & B.T.C., B.E.,B.P. & $50-1700 m$ \\
\hline K. humboldtiana & Jun-Sep & Invierno & $\begin{array}{l}\text { M.X., B.T.C., B.E., } \\
\text { B.T.P., PAST. }\end{array}$ & $0-2200 m$ \\
\hline K. johnstonii & May-Jul & Ags-Oct & B.T.C & $350-450 m$ \\
\hline K. latifolia & Mar-May & Jul-Ags & B.T.C. & $1300-1800 \mathrm{~m}$ \\
\hline K. mollis & Jun-Jul & Ags-Sep & M.X., B.J. & $1000-2000 m$ \\
\hline K. parvifolia & Jun-Jul & Sep-Ene & B.T.C. & $20-150 m$ \\
\hline K. rzedowskii & Abr-Ags & Sep-Oct & B.T.C. & $1000-1700 \mathrm{~m}$ \\
\hline K. subcordata & May-Jun & Jul-Sep & M.X. & $1500-2000 \mathrm{~m}$ \\
\hline K. tehuacana & Jun-Jul & Sep-Nov & M.X. & $1400 \mathrm{~m}$ \\
\hline K. umbellata & Feb-Abr & Sep-Nov & B.T.C. & $850-1200 m$ \\
\hline K. venturae & May-Jun & Ags-Sep & B.T.C. & $950-1530 \mathrm{~m}$ \\
\hline
\end{tabular}

* B.T.C. = Bosque Tropical Caducifolio; B.T.P. = Bosque Tropical Perenifolio; B.E. = Bosque de Encino;

B.P. = Bosque de Pinos; B.J. = Bosque de Juniperos; M.X. = Matorral Xerófito; PAST. = Pastizal.

Fuente: Fernandez 1988, 1992, 1993; Standley 1928 


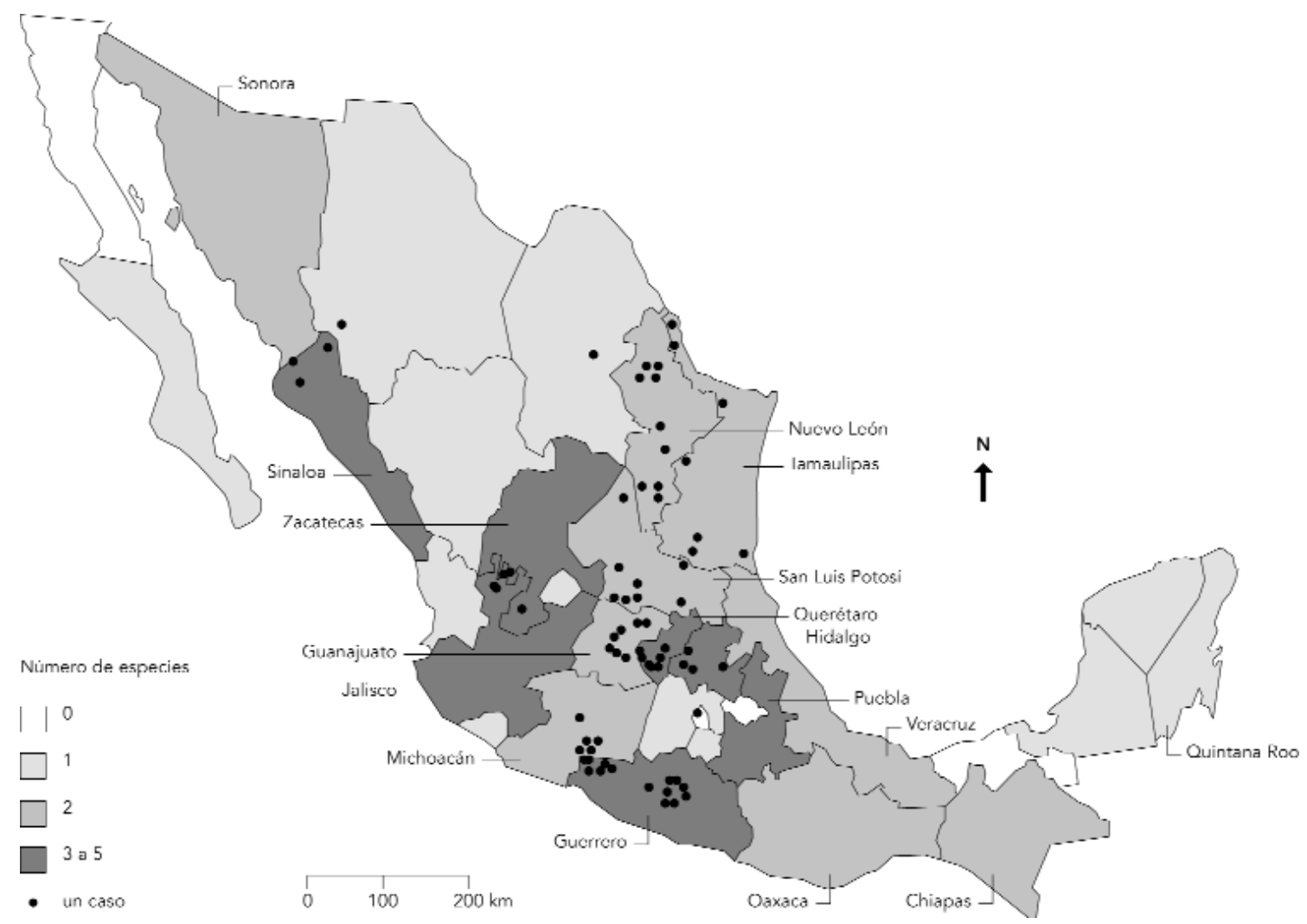

Elaboró: M. E. Arreola Nava.

Fuente: Fernández, 1992; Dirección General de Epidemiología, Secretaría de Salud.

Figura 2

Casos de intoxicación por Karwinskia según grupos de edad, 1990-1994.

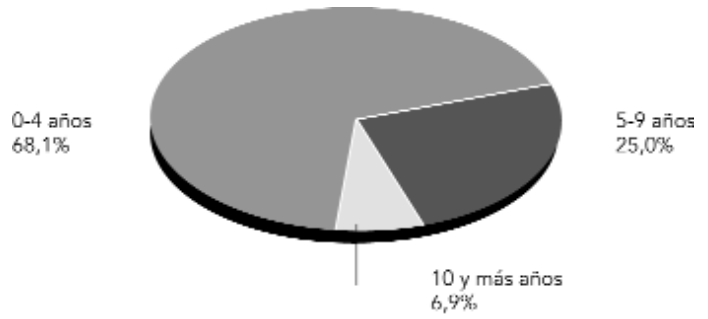

Fuente: Dirección General de Epidemiología, Secretaría de Salud. de vegetación se desarrollan las especies de $K$. humboldtiana, K. mollis, K. subcordata y K. tehuacana, como propias del matorral. El 26,4\% (19 casos) corresponde al Bosque Tropical Caducifólio, con especies de Karwinskia calderonii, K. humboldtiana, K. johnstonii, K. latifolia, K. parvifolia, K. rzedowskii, K. umbellata y K. venturae. La distribución de los casos con respecto al clima mostró que el mayor número se presenta en el grupo de climas Secos BS: 57 casos $(79,2 \%)$; de estos, el clima Semiárido BS1 concentra 44 de los casos $(61,1 \%)$. Mientras que, en localidades correspondientes al clima Árido BS0, se dieron 13 casos (18,1\%). Se pudo observar que, en altitudes de 0 a 500 metros sobre el nivel del mar, se presentaron 24 casos (33,3\%); de 501 a 1000 metros, 15 casos $(20,8 \%)$; de 1501 a 2000 metros, 22 casos (30,6\%). Es importante resaltar que el $50 \%$ de los casos no re- 


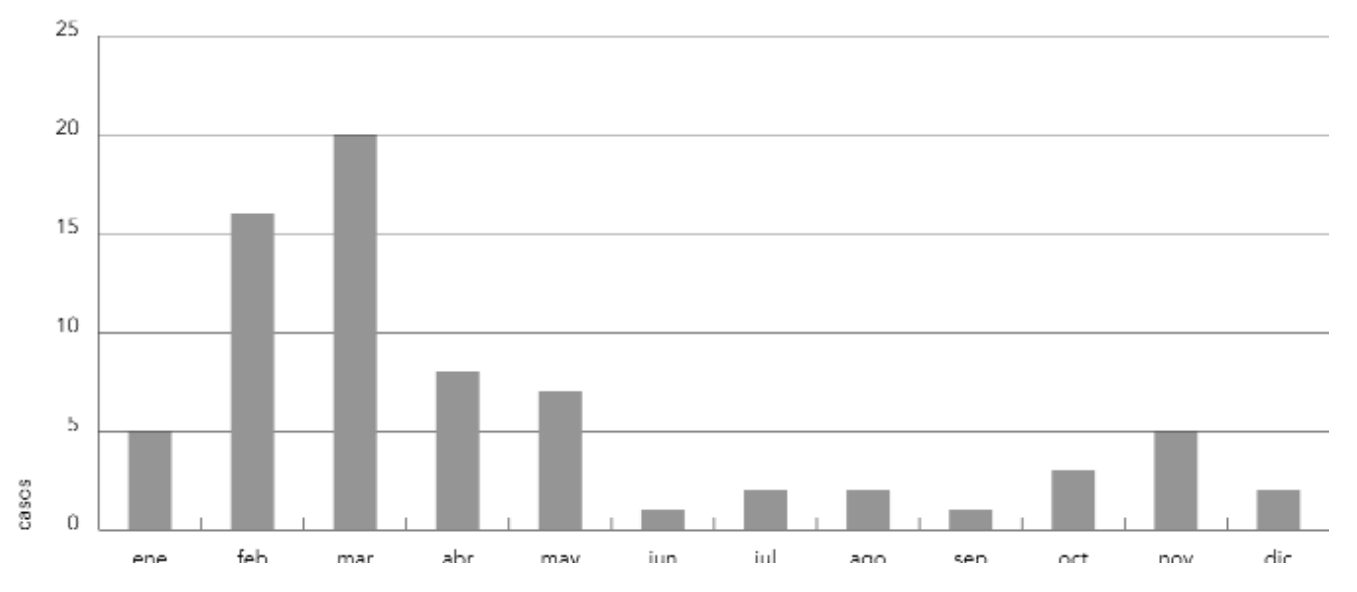

Fuente: Dirección General de Epidemiología, Secretaría de Salud.

basan los mil metros de altitud, por lo que son las partes bajas de las sierras que coinciden con la mayor presencia de especies como la $K$. humboldtiana, K. johnstonii, K. calderonii y $K$. parvifolia.

Muchos de los casos (56,3\%) se presentaron en municipios que tienen alto grado de ruralidad, con niveles de escolaridad bajos (79,2 \%), o bien en municipios con bajos niveles de bienestar (68,1 \%) (Inegi, 1993) (Tabla 2).

\section{Conclusiones}

La localización de los casos de intoxicación coincide con la ubicación de plantas de la Karwinskia que son exclusivas en el país, tales como K. mollis, K. rzedowskii, K. parvifolia, $K$. venturae, presentes en los estados de Sinaloa, San Luis Potosí, Michoacán, Jalisco, Zacatecas, Tamaulipas y Guerrero. La intoxicación por Karwinskia es un problema de salud de carácter regional, y las poblaciones susceptibles de presentar casos de intoxicación en México, además de la presencia de la planta, son las que presentan las siguientes características: Vegetación de Matorrales áridos y semiáridos, Bosque Tropical Caducifólio y zonas perturbadas (agricultura); Climas Secos, con la particularidad de escasez de agua para otros grupos de climas; Suelos feozem, vertisol y litosol, cuya característica principal de estos tres es que son de amplia distribución en el país y permi-
Tabla 2

Principales aspectos geográficos en los casos de intoxicación por Karwinskia en México, 1990-1994.

\begin{tabular}{|c|c|c|}
\hline Aspectos geográficos & Casos & Porcentaje \\
\hline \multicolumn{3}{|l|}{ Climas } \\
\hline BS Secos & 57 & $79,2 \%$ \\
\hline \multicolumn{3}{|l|}{ Vegetación } \\
\hline Matorral Árido y Semiárido & 30 & $41,7 \%$ \\
\hline Bosque Tropical Caducifolio & 19 & $26,4 \%$ \\
\hline \multicolumn{3}{|l|}{ Suelos } \\
\hline Feozem & 19 & $26 \%$ \\
\hline Vertisol & 14 & $19,4 \%$ \\
\hline \multicolumn{3}{|l|}{ Altitudes } \\
\hline De 0 a $500 \mathrm{mts}$ & 24 & $33,3 \%$ \\
\hline De 501 a $1000 \mathrm{mts}$ & 17 & $23,6 \%$ \\
\hline De 1001 a $1500 \mathrm{mts}$ & 0 & $00,0 \%$ \\
\hline De 1501 a $2000 \mathrm{mts}$ & 23 & $31,9 \%$ \\
\hline \multicolumn{3}{|l|}{ Aspectos sociales } \\
\hline \multicolumn{3}{|l|}{ Ruralidad } \\
\hline Alta & 47 & $65,3 \%$ \\
\hline \multicolumn{3}{|l|}{ Escolaridad } \\
\hline De 3 a 6 años aprobados & 57 & $79,2 \%$ \\
\hline \multicolumn{3}{|l|}{ Niveles de bienestar } \\
\hline Bajos & 49 & $68,1 \%$ \\
\hline
\end{tabular}

Fuente: Cartas temáticas 1:50000, Niveles de Bienestar 1993. Instituto Nacional de Estadística, Geografía e Informática. 
ten, entre otros tipos de vegetación, el desarrollo de matorrales áridos y semiáridos. Altitudes menores, que van de los 0 a los 1000 m.s.n. m., en declives montañosos o de ladera. Características de población de alta ruralidad y bajos niveles de bienestar.

Zonas específicas, como la depresión del Río Balsas y del Río Balsas-Mezcala (Michoacán y Guerrero), presentan las características mencionadas, además de cinco de las once especies de Karwinskia en el país. Otra zona es la comprendida por los estados de San Luis Potosí

\section{Agradecimientos}

Dr. Raúl Montesano Castellanos y Dr. Miguel Angel Vázquez Guerrero, Dirección General de Epidemiología. Dr. M. Victoria Bermúdez, Departamento de Farmacología y Toxicología de la Facultad de Medicina de la Universidad Autónoma de Nuevo León. Biol. Teresa Germán, Herbario Nacional de la Universidad Nacional Autónoma de México (MEXU). Instituto de Botánica de la Universidad de Guadalajara.

\section{Referencias}

BERMUDEZ, R. V.; LOZANO, M. F.; TAMEZ, R.; DIAZ, C. G. \& PIÑEYRO, L. A., 1995. Frecuencia de intoxicación con Karwinskia humboldtiana en México. Salud Pública de México, 37:57-62.

BERMUDEZ, R. M. V., 1989. Toxicidad Aguda de la Karwinskia humboldtiana en Diferentes Especies de Animales. Tesis de Doctorado, Monterrey: Universidad Autónoma de Nuevo León.

CARRADA-BRAVO, T.; LOPEZ, L. H.; VAZQUEZ, A. G \& LEY, L. A., 1983. Brote epidémico de polirradiculoneuritis por tullidora (Karwinskia humboldtiana). Boletín Médico del Hospital Infantil de México, 40:139-147.

CASTILLO-NAJERA, F., 1918. Contribución al estudio de las parálisis tóxicas. Un envenenamiento colectivo por "tullidora”. Memorias del V Congreso Médico Mexicano, pp. 240-243, Puebla: Instituto Médico Nacional.

ESCOBAR, I. A. \& NIETO, D., 1965. Aspectos neuropatológicos de la intoxicación con Karwinskia humboldtiana estudio experimental. Gaceta Médica de México, 2:163-177.

FERNANDEZ, N. R., 1988. Tres especies nuevas de Karwinskia (Rhamnaceae) de México. Acta Botánica Mexicana, 2:11-20.

FERNANDEZ, N. R., 1992. Nombres comunes, usos y distribución geográfica del género Karwinskia (Rhamnaceae) en México. Anales del Instituto de Biología, UNAM, 63:1-23.

FERNANDEZ, N. R., 1993. La Familia Rhamnaceae en México. Tesis de Doctorado, México, D.F.: Instituto Politécnico Nacional, Escuela Nacional de Ciencias Biológicas (municipios de Matehuala, Ríoverde, San Nicolás Tolentino), Querétaro (municipios de Querétaro, Toliman, Ezequiel Montes, Corregidora), Hidalgo (municipios de Huichapan, Tlaxcoapa, Zimapan), Guanajuato (municipios de Tierra Blanca, Santa Catarina, Allende).

Las relaciones encontradas, con las variables de ruralidad, nivel de escolaridad y niveles de bienestar, sugieren la necesidad de ampliar las investigaciones al respecto sobre aspectos sociales, culturales y económicos que intervienen en la intoxicación.
GARZA, O. L.; GIN, C. H.; ACOSTA, D.; TORRES, A. O. \& PIÑEYRO, L. A., 1992. Toxicity assessment of toxins t-514 and t-544 of buckthorn (Karwinskia humboldtiana) in primary skin and liver cell cultures. Toxicology, 73:191-201.

HERNANDEZ, C. A. \& MUÑOZ, M. E. J., 1982. Distal reduction of the conduction velocity of a-axons in Tullidora (buckthorn) neuropathy. Experimental Neurology, 82:335-343.

HERNANDEZ, C. A. \& MUÑOZ, M. E. J., 1984. Axonto-axon transmission in tullidora (buckthorn) neuropathy. Experimental Neurology, 84:533-548.

INEGI (Instituto Nacional de Estadística, Geografía e Informática), 1993. Niveles de bienestar en México. México, D.F.: Secretaría de Programación y Presupuesto.

PADRON, P. F., 1951. Estudio clínico experimental de la parálisis por Karwinskia humboldtiana (tullidora) en niños. Gaceta Médica de México, 81:300311.

PUERTOLAS, M. M.; NAVA, J. O.; MEDINA, L. H.; LOPEZ, O. F. \& OYERVIDES, P. J., 1984. Polirradiculoneuritis por Karwinskia humboldtiana informe de seis casos. Revista Médica del IMSS, 22:25-27.

STANDLEY, P. C., 1928. Trees and Shrubs of Mexico. U.S. vol. 23. Washington, D.C.: Smithsonian Institute.

WAKSMAN, N.; MARTINEZ, L. \& FERNANDEZ, R., 1989. Chemical and toxicological screening in genues Karwinskia (Mexico). Revista Latinoamericana de Química, 20:27-32. 